\title{
Intestinal absorption of linoleic acid in experimental renal failure
}

\author{
BY M. V. PAHL, A. BARBARI, N. D. VAZIRI, D. HOLLANDER, M. YAZDANI, \\ J. TRAN AND R. KAN \\ Division of Nephrology, Department of Medicine, University of California at Irvine, Irvine, \\ California 92668, USA
}

(Received 23 July 1990 - Accepted 5 March 1991)

\begin{abstract}
Linoleic acid (LA) transport in rats with experimental short-term and long-term renal failure (RF) was compared with that of sham-operated normal animals on liberal food intake and pair-fed animals. The perfusions in vivo and incubations in vitro were conducted using a micellar solution containing a wide range of LA concentrations. Both absorption in vivo and uptake in vitro of LA were significantly reduced in animals with short-term RF. Lipid extraction and separation by thin-layer chromatography revealed a marked LA trapping as trilinolein (TL) in the perfused intestinal tissue in the short-term RF group. The esterification process, as defined by the rate of $L A$ incorporation into $T L$, was moderately reduced in short-term RF animals. The thickness of the unstirred water layer showed no significant difference among the groups studied. In contrast, animals with long-term RF exhibited normal absorption of LA in vivo at all concentrations tested. In conclusion, LA absorption is reduced in short-term RF and restored in long-term RF. Several steps including LA transport into and TL transport out of the enterocyte and the esterification process were impaired in short-term RF. These changes are not due to alteration in the unstirred water layer, anorexia, weight loss or a rapid effect of uraemic chemical environment or circulatory factors.
\end{abstract}

Renal failure: Fatty acid absorption: Intestinal transport

Linoleic acid (LA) is a long-chain polyunsaturated dietary essential fatty acid whose deficiency in humans can lead to skin disorders, growth retardation, increased platelet aggregation, diminished prostaglandin synthesis and possibly increased incidence of arteriosclerotic cardiovascular disease (Vergroesen, 1977). Experimental and clinical uraemia has been shown to be associated with impaired intestinal absorption of various nutrients including iron, amino acids, dipeptides, riboflavin, vitamin $\mathrm{D}_{3}$ and folate (Delano et al. 1977; Sterner et al. 1982; Vaziri et al. 1983; Said et al. 1984). However, the possible effect of renal failure on the intestinal absorption of LA has not been previously investigated. We investigated the intestinal transport of this biologically important essential fatty acid in experimental renal failure $(\mathrm{RF})$ in the rat.

\section{MATERIALS AND METHODS}

Male Sprague-Dawley rats weighing 250-280 g, fed on Purina rat chow and water ad lib., were randomly assigned to short-term or long-term RF and control groups. The former groups were included to differentiate the possible effects of duration of renal insufficiency on the rate of LA absorption. Animals placed in the RF group underwent a concurrent right nephrectomy and left two-thirds nephrectomy. In animals assigned to the short-term RF group the remaining tissue was partly cauterized. The procedure was performed extraperitoneally through a single dorsal incision. Strict haemostasis and aseptic measures were observed. All surgical procedures were conducted under general anaesthesia using an intraperitoneal injection of sodium pentobarbital $(65 \mathrm{mg} / \mathrm{kg})$. A pair-fed (PF) group was 
included to control for uraemia-induced anorexia, reduced nutrient intake and weight loss. Pair-feeding was accomplished by limiting the daily food available to the PF animal to that consumed by its RF counterpart during the preceding $24 \mathrm{~h}$. Animals placed in the normal control (NL) group underwent surgery with exposure and manipulation of the kidneys without performing the nephrectomies. The short-term RF and control groups were allowed a 2-week post-operative recuperation period before the absorption experiments, while the long-term RF and their controls were studied 5-6 weeks post-operatively. Serum creatinine was determined in tail venous blood on the day before the absorption experiments using a kit purchased from Sigma Chemical Co., St Louis, MO.

Materials. $\left[{ }^{14} \mathrm{C}\right]$ LA (New England Nuclear, Boston, MA) with a specific activity of $52.6 \mathrm{mCi} / \mathrm{mmol}$ was used as a tracer compound, $\left[{ }^{3} \mathrm{H}\right]$ inulin (ICN Pharmaceuticals Inc., Irvine, CA) with a specific activity of $60 \mathrm{mCi} / \mathrm{g}$ was used as a non-absorbable marker and ${ }^{14} \mathrm{C}$-labelled warfarin (Amersham Co., Arlington Heights, IL) with a specific activity of $50-60 \mathrm{mCi} / \mathrm{mmol}$ was used as a probe to measure the unstirred water layer. Purified sodium taurocholate (used as surfactant), unlabelled LA and its corresponding monoglyceride were purchased from Sigma Chemical Co., St Louis, MO. A micellar solution of Krebs phosphate buffer ( $\mathrm{pH} \mathrm{6.5)} \mathrm{containing} 10 \mathrm{~mm}$-sodium taurocholate, trace amounts of $\left[{ }^{14} \mathrm{C}\right] \mathrm{LA}$ and $\left[{ }^{3} \mathrm{H}\right]$ inulin, unlabelled LA $(42-4200 \mu \mathrm{M})$ and in some experiments monolinolein (ML) with a concentration ratio (LA:ML) of $2: 1$, was prepared by sonication of $70 \mathrm{~W}$ for 5 min at $4^{\circ}$.

Absorption studies in vivo. Under diethyl ether anaesthesia, the abdomen was opened by a midline incision. An inflow polyethylene cannula was inserted into the jejunum $50 \mathrm{~mm}$ distal to the ligament of Treitz and an outflow L-shaped glass cannula was introduced $200 \mathrm{~mm}$ distal to the inflow cannula. The cannulas were secured by ligating between parallel end-vessels to avoid obstructing blood and lymphatic vessels. The intestinal segment was then placed in the peritoneal cavity, the abdomen was closed and the animal was placed in a restraining cage. The inflow cannula was then connected to a reservoir containing $50 \mathrm{ml}$ perfusate, which was continually stirred with a magnetic stirrer. A totally occlusive roller pump (Buchler Instruments, Inc., Fort Lee, NJ) was employed to pump the perfusate from the reservoir through the inflow cannula, and the outflow cannula was allowed to drain by gravity back into the reservoir. The animal's body temperature was closely monitored and kept at $37^{\circ}$ using a forced-air heating device and a thermostatic temperature controller (Thermistemp Model 74, Yellow Spring, $\mathrm{OH}$ ) with a rectal probe. Experiments were performed under subdued light, and LA supplies were protected from light exposure using aluminium foil in order to avoid denaturation. The rate of LA absorption was determined from the rate of its disappearance from the perfusate and $\left[{ }^{3} \mathrm{H}\right]$ inulin was used as a nonabsorbable marker to correct for fluid shifts (Hollander, 1981). The following equation was employed: $A_{t}=A_{0}\left(I_{0} / L_{0} \cdot L_{t} / I_{t}\right)$, where $A_{t}$ and $A_{0}$ represent the amount of LA in the perfusate at times $t$ and 0 and $L_{0}$ and $L_{t}$ equal the concentration of labelled LA at times 0 and $t$, and $I_{0}$ and $I_{t}$ stand for the concentrations of radiolabelled inulin at times 0 and $t$ respectively. Samples were obtained from the perfusate immediately before the onset of the $40 \mathrm{~min}$ perfusion and every $10 \mathrm{~min}$ thereafter. The absorption studies were conducted using two different concentrations of LA, i.e. low concentration $(840 \mu \mathrm{M})$ and high concentration $(4200 \mu \mathrm{M})$. At the conclusion of the study, the animal was killed with an overdose of diethyl ether and the perfused intestinal segment was removed, washed and hung vertically for $24 \mathrm{~h}$ with a $10 \mathrm{~g}$ weight attached to its most dependent portion. The dry length of the intestine was then noted and the segment was cut into three pieces of equal length, further dried at $60^{\circ}$ and crushed and solubilized with BTS-450 (Beckman, Fullerton, CA) for determination of the residual tissue radioactivity. In order to assess the rate of intestinal trilinolein (TL) synthesis and the distribution of LA among different glyceride 
fractions, the experiments performed in vivo were repeated and the perfused intestinal segments were processed for lipid extraction and separation by thin-layer chromatography (TLC) as described in the following sections.

Studies in vitro. Rats were killed by cervical dislocation. The proximal jejunum was removed, washed with iced Krebs phosphate buffer and divided into several segments of equal lengths. Each segment was everted, ligated at both ends and incubated in $6 \mathrm{ml}$ of the micellar solution. Incubations were carried out at $37^{\circ}$ in a shaking water-bath (80 oscillations $/ \mathrm{min}$ ) for $10 \mathrm{~min}$. Pre-incubation samples were withdrawn in duplicate from the solution. At the conclusion of the incubation period, sacs were removed and immediately rinsed in iced taurocholate solution ( $1 \mathrm{~mm}$ ) for $15 \mathrm{~s}$ and gently blotted on paper towels in order to remove any solution adhering to the sac's surface. Sacs were dried in an oven at $60^{\circ}$ for $48 \mathrm{~h}$, weighed in the dry state, crushed and solubilized with BTS-450 (Beckman, Fullerton, CA) for radioactivity determination. $\left[{ }^{3} \mathrm{H}\right]$ inulin was used as non-absorbable marker to determine the volume of incubation fluid remaining adherent to the sac surface and to measure the net mucosal uptake of LA. The latter was calculated by subtracting the amount present in the adhering mucosal fluid from the total uptake (Chow \& Hollander, $1979 a$ ). The experiments performed in vitro were repeated with sacs pre-incubated for $10 \mathrm{~min}$ in an iced $840 \mu \mathrm{M}$-LA micellar solution $\left(0-1^{\circ}\right)$, a temperature which is known to inhibit esterification reversibly (Bennet Clark et al. 1973; Ockner \& Isselbacher, 1974). Sacs were then further re-incubated in Krebs phosphate buffer (pH 6.5) at $37^{\circ}$ for 1,3 and 5 min. The rate of subsequent conversion of the absorbed LA to $\mathrm{TL}$ at $37^{\circ}$ was considered as the rate of LA esterification. Since transmural absorption of long-chain fatty acids requires an intact lymphatic system which is lacking in the preparation in vitro, the results merely reflect mucosal uptake (Chow \& Hollander, 1979a).

Lipid extraction and separation. Intestinal segments obtained after the experiments in vivo and in vitro were immediately placed in cold methanol at $-20^{\circ}$ to prevent further esterification (Ockner et al. 1972). Tissues were then processed for lipid extraction (Kates, 1975) and separation by TLC (Brown \& Johnston, 1962). A mixture of pure components containing TL, dilinolein (DL), ML and LA was co-chromatographed simultaneously on the same plate. Once identified under u.v. light, specific spots corresponding to various glyceride subfractions were scraped and transferred to separate scintillation vials, then solubilized with BTS-250. Similarly, portions of lipid extracts were withdrawn and processed for radioassays. Negligible amounts of radioactivity were found in the tissue precipitate after final lipid extraction, and the percentage of radioactivity recovered from the chromatogram ranged from 86 to $98 \%$, with a mean of $92 \%$.

Unstirred water layer. The unstirred water layer was measured using a single-pass perfusion method with ${ }^{14} \mathrm{C}$-labelled warfarin as described by Anderson et al. (1988). $\left[{ }^{3} \mathrm{H}\right]$ inulin $(100-500 \mathrm{mCi} / \mathrm{g})$ was used as a non-absorbable marker.

Radioisotope counting. Samples withdrawn from perfusates, incubation media and lipid extracts as well as tissue homogenates and silica-gel scraping were mixed with appropriate amounts of liquid-scintillation cocktail (rPi, Mount Prospect, Ill.) and the radioactivity was determined by double-isotope counting carried out to $\pm 0.5 \%$ sigma error using a liquidscintillation counter (Beckman LS-9000) with an automatic quench calibration programme at ambient temperature.

Statistical analysis. Where indicated Student's $t$ test and ANOVA (Draper \& Smith, 1966) were used for analysis of the data which are given as means with their standard errors.

RESULTS

As expected, short-term RF and PF animals exhibited comparable reduction in bodyweights, while the NL animals gained from 5 to $25 \mathrm{~g}$ during the 2-weeks period before the 
absorption experiments. A reduction in the dry intestine weight per unit length $(30-32 \%)$ was observed in short-term RF and PF animals when compared with the NL control group (g/30 mm; short-term RF 0.032 (SE 0.001), PF 0.032 (SE 0.001), NL controls 0.055 (SE $0.001) ; P<0.01$, short-term RF and PF v. NL controls). In contrast to the animals with short-term RF, animals with long-term RF (and their PF counterparts) showed a moderate increase in body-weight, although not as much as that seen in the NL control counterparts. Intestine weight $(\mathrm{g} / 30 \mathrm{~mm})$ in the long-term RF group $(0.045$ (SE 0.003)) was not significantly different from either the corresponding PF group (0.042 (SE 0.003)) or the normal control group (0.051 (SE 0.003)).

Absorption in vivo. Serum creatinine levels were significantly higher in both short-term and long-term RF groups than those found in their corresponding PF and NL control groups (Tables 1 and 2). Intestinal absorption of LA at low and high perfusate concentrations was significantly reduced in the short-term RF group when compared with the NL group. The amount of residual $\left[{ }^{14} \mathrm{C}\right] \mathrm{LA}$ remaining in the mucosa of the perfused segment at the conclusion of the experiment in the short-term RF group was nearly twice that found in the NL control group at both concentrations. Despite exhibiting a weight loss comparable with that of the short-term RF group, the absorption rate in the PF group was significantly higher $(P<0.02)$ than that found in the short-term RF group and not significantly different from that found in the ad lib.-fed NL group. In addition, there was no difference between $\mathrm{PF}$ and $\mathrm{NL}$ groups with regard to the residual $\left[{ }^{14} \mathrm{C}\right] \mathrm{LA}$ remaining in the perfused mucosal tissue. The findings are shown in Table 1 . The experiments performed in vivo were repeated using low and high LA concentrations with ML at concentrations of $840 \mu \mathrm{M}: 420 \mu \mathrm{M}$ and $4200 \mu \mathrm{M}: 2100 \mu \mathrm{M}$ respectively. Results obtained from lipid extraction and separation by TLC (Tables 3 and 4) indicated that most of the residual $\left[{ }^{14} \mathrm{C}\right] \mathrm{L}$ A found in the mucosa of the perfused segment was incorporated into TL in both short-term RF and NL groups, but the amount of tissue TL in short-term RF animals was twice that found in the NL animals.

In contrast to the short-term model, the rates of LA absorption in animals with longterm RF were comparable with those found in their NL and PF controls, at all concentrations tested. Furthermore, there was no evidence of increased residual $\left[{ }^{14} \mathrm{C}\right] \mathrm{LA}$ remaining in the perfused intestinal segment of the long-term RF animals. The findings are shown in Table 2.

Unstirred water layer. Absorption of ${ }^{14} \mathrm{C}$-labelled warfarin, the probe used to measure the unstirred water layer, was found to be comparable among the three groups studied. Similarly, there was no difference in the measurement of the unstirred water layer among the three groups ( $\mu \mathrm{m}$ : short-term RF 392 (SE 191), NL 476 (SE 122), PF controls 387 (SE 115)).

Uptake and esterification in vitro. The results obtained for the uptake studies performed in vitro for low concentrations $(42-1260 \mu \mathrm{M})$ of LA are shown in Fig. 1. Maximum velocity $\left(V_{m}\right)$ and apparent $K_{t}$ were obtained using the Lineweaver-Burk plot. Saturation of uptake was observed with increasing LA concentrations in the incubation medium in both groups. However, the rate of LA uptake was consistently lower in short-term RF animals than in control animals. Similarly, the calculated values for $K_{t}$ and $V_{m}$ were lower in the short-term RF group ( $726 \mu \mathrm{M}$ and $0.230 \mu \mathrm{mol} / 100 \mathrm{~mm}$ per $10 \mathrm{~min}$ ) when compared with those of the control animals $(1067 \mu \mathrm{M}$ and $0.320 \mu \mathrm{mol} / 100 \mathrm{~mm}$ per $10 \mathrm{~min})$. In addition, the rate of LA uptake at high concentration $(4200 \mu \mathrm{M})$ was significantly lower $(0.498$ (SE 0.044) $\mu \mathrm{mol} / 100 \mathrm{~mm}$ per $10 \mathrm{~min})$ in the short-term RF group $(P<0.02)$ than in the control animals $(0.598$ (SE 0.088$) \mu \mathrm{mol} / 100 \mathrm{~mm}$ per $10 \mathrm{~min}$ ). The rates of LA uptake in vitro at a concentration of $840 \mu \mathrm{M}$ in the PF group were not significantly different from those found in the NL animals $(0.17$ (SE 0.01) $\mu \mathrm{m} / 100 \mathrm{~mm}$ per $10 \mathrm{~min} v .0 .17$ (SE 0.02$) \mu \mathrm{mol} / 100 \mathrm{~mm}$ per 


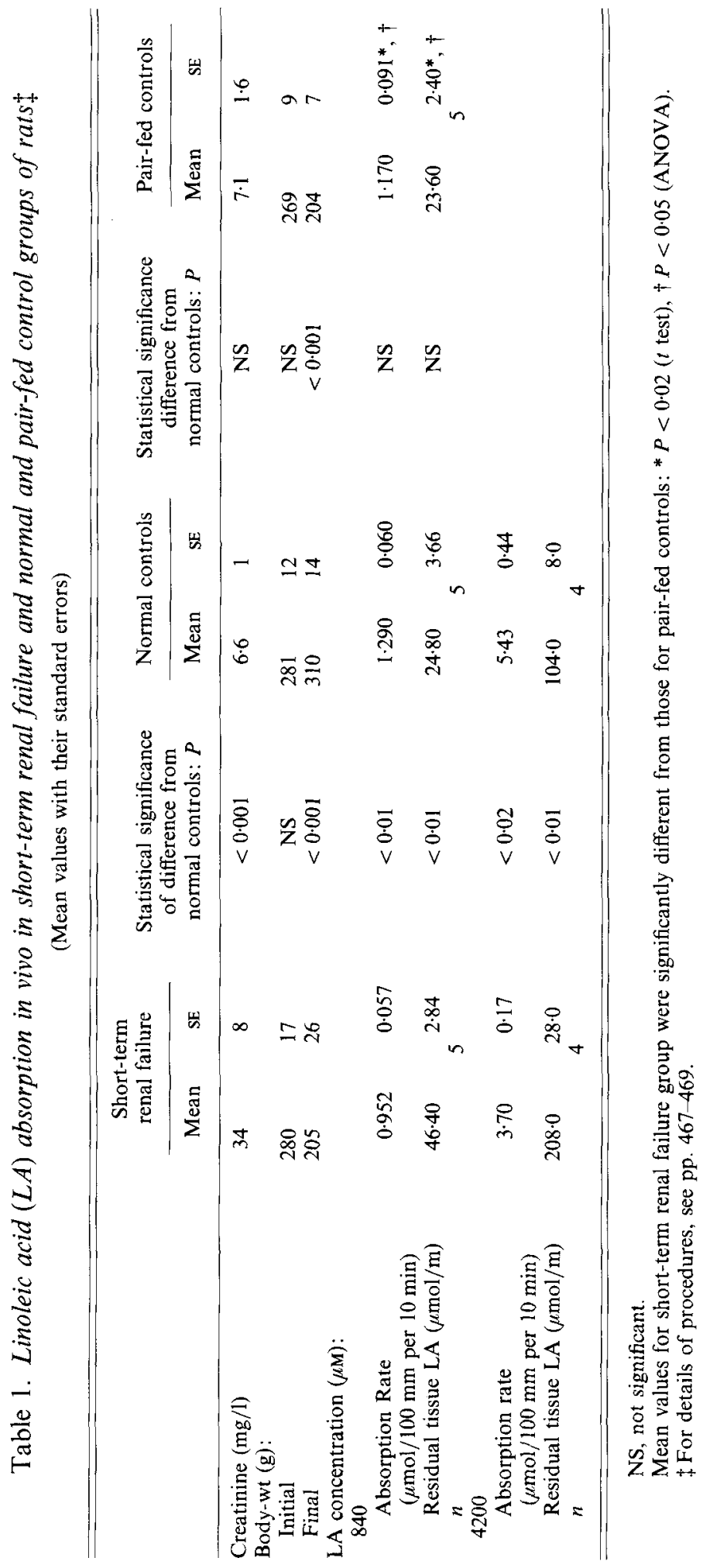


M. V. PAHL AND OTHERS

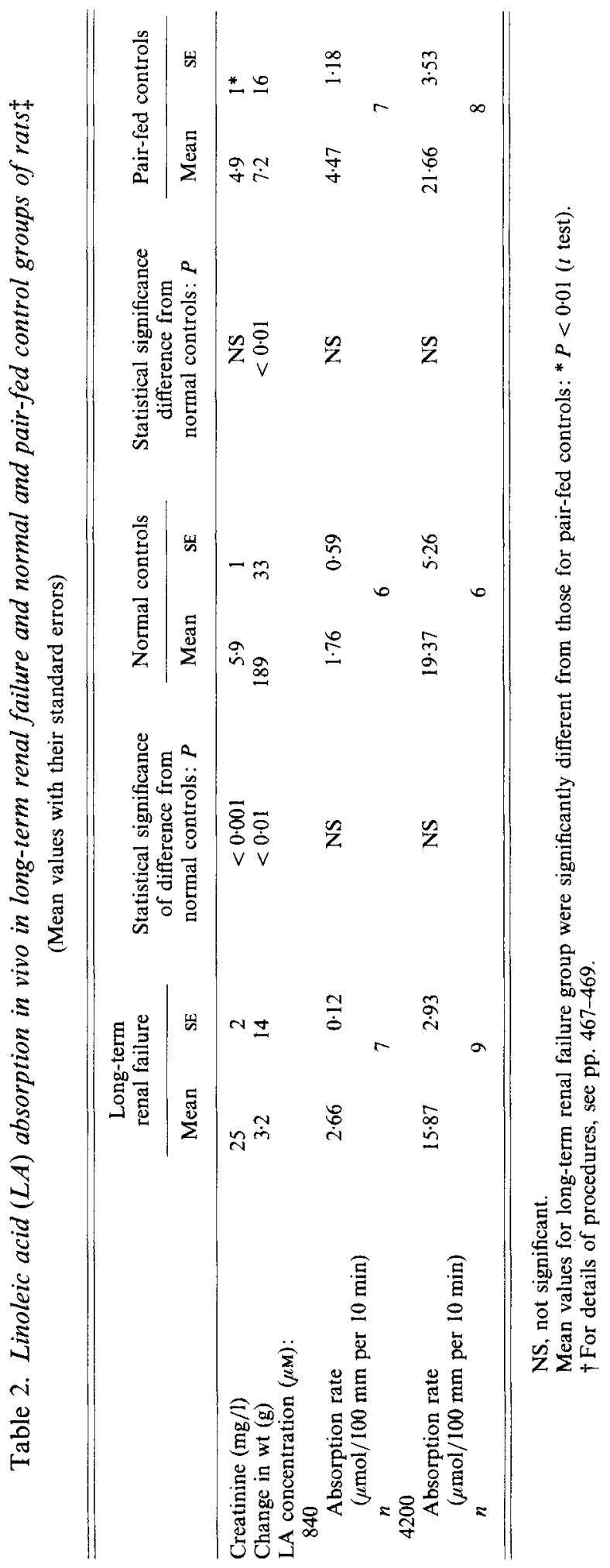


Table 3. Distribution in vivo of linoleic acid $(L A)$ among glyceride fractions at low $L A$ concentration in the perfusion solution in short-term renal failure and normal control groups of rats*

(Results showing the absorption rate and the amount of residual tissue LA at a concentration of LA:ML of $840 \mu \mathrm{M}: 420 \mu \mathrm{M}$. Different glyceride fractions were obtained by thin-layer chromatography. Each glyceride fraction is expressed as the amount of incorporated LA. Values are means with their standard errors for six animals per group)

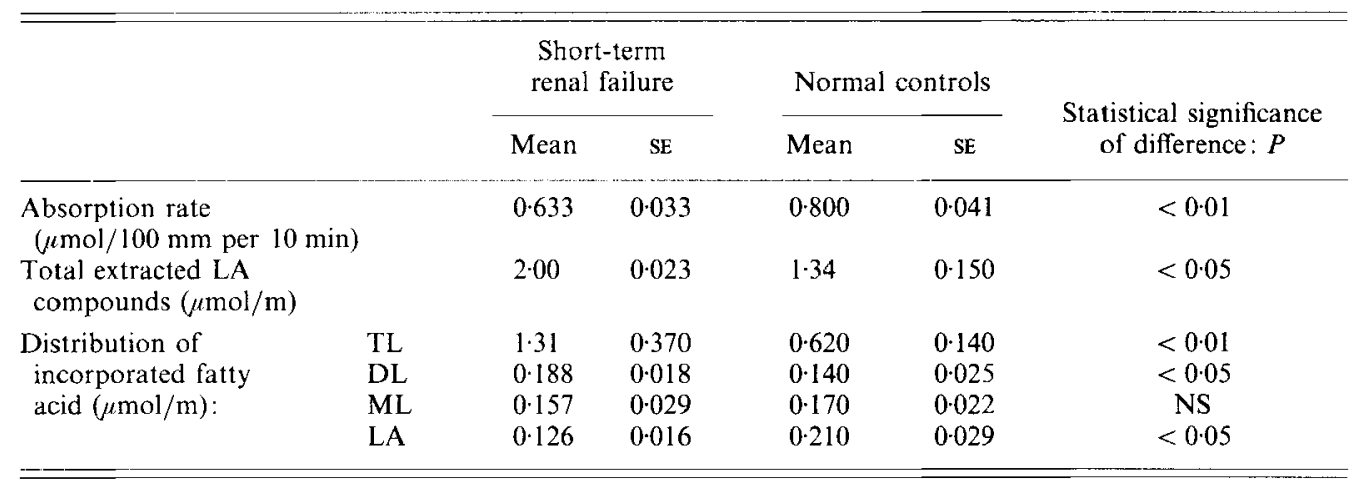

TL, trilinolein; DL, dilinolein; ML, monolinolein; NS, not significant.

* For details of procedures, see pp. 467-469.

Table 4. Distribution in vivo of linoleic acid $(L A)$ among different glyceride fractions at high $L A$ concentration in the perfusion solution in short-term renal failure and normal control groups of rats*

(Results showing the absorption rate and the amount of residual tissue LA at a concentration of LA : MA of $4200 \mu \mathrm{M}: 2100 \mu \mathrm{M}$. Different glyceride fractions were obtained by thin-layer chromatography. Each glyceride fraction is expressed as the amount of incorporated LA. Values are means with their standard errors for six animals per group)

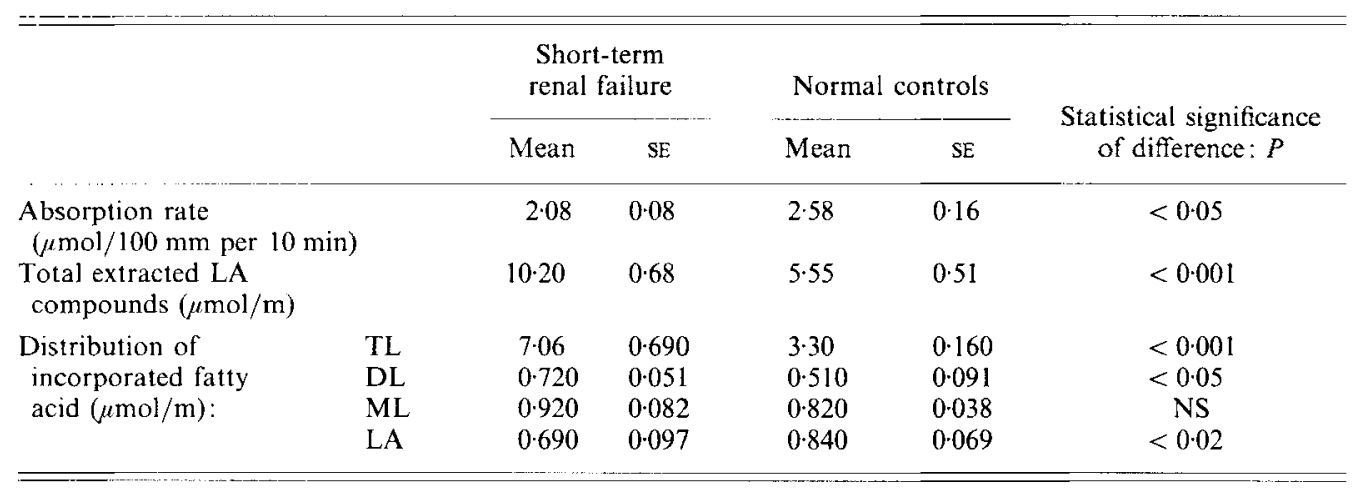

TL, trilinolein; DL, dilinolein; ML, monolinolein; NS, not significant.

* For details of procedures, see pp. 467-469.

10 min respectively). In order to separate the effect of renal failure on the mucosal uptake from that on the esterification process, pre-incubations in vitro were carried out for $10 \mathrm{~min}$ at $0-1^{\circ}$, which reversibly inhibits esterification. The same intestinal sacs were then immediately re-incubated in Krebs phosphate buffer at $37^{\circ}$ for 1,3 and 5 min. The rates of LA uptake in the cold were comparable in both groups $(\mu \mathrm{mol} / 100 \mathrm{~mm}$ per $10 \mathrm{~min} ; 0 \cdot 106$ (SE 0.010) v. 0.106 (SE 0.011) in short-term RF and NL control tissues respectively). 


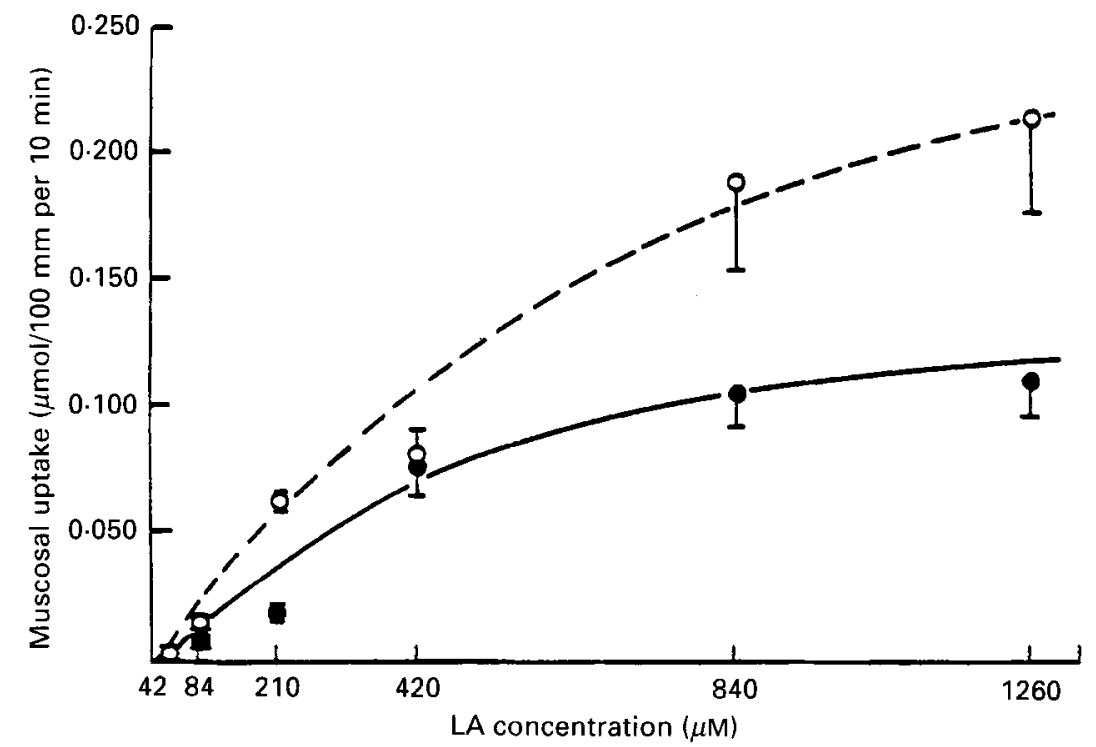

Fig. 1. Relationship between linoleic acid (LA) concentration and its uptake in vitro in short-term renal failure (O) and normal $(O)$ control groups of rats. Each point represents the mean rate of uptake at a specific concentration with their standard errors represented by vertical bars for five to seven observations. For details of procedures, see pp. 467-469. Short-term renal failure: $V_{m} 0.230 \mu \mathrm{M} / 100 \mathrm{~mm}$ per $10 \mathrm{~min}, K_{t} 726 \mu \mathrm{M}$, normal control: $V_{m} 0.320 \mu \mathrm{M} / 100 \mathrm{~mm}$ per $10 \mathrm{~min}, K_{t} 1067 \mu \mathrm{M}$, where $V_{m}$ is maximum velocity and $K_{t}$ is the Michaelis constant.

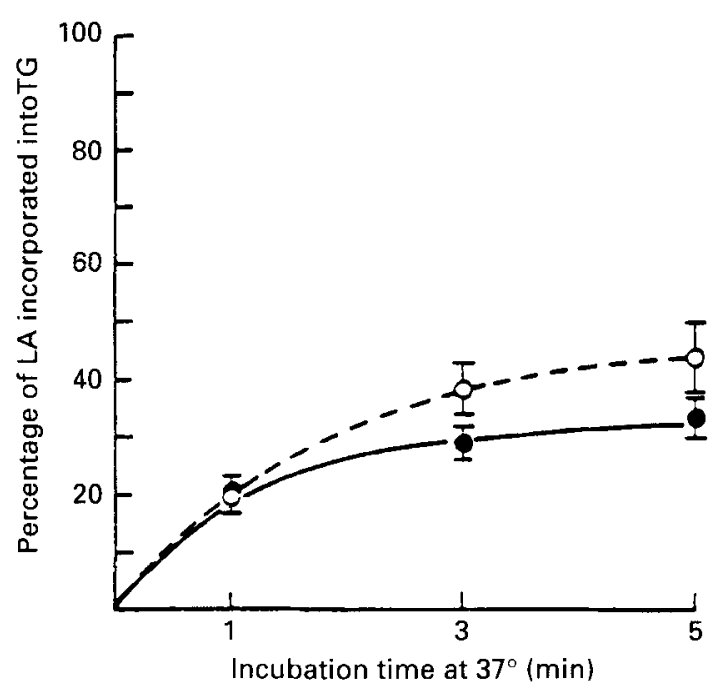

Fig. 2. Linoleic acid (LA) incorporation in vitro into triacylglycerol (TG) in short-term renal failure (O) and normal $(O)$ control groups of rats at $37^{\circ}$ after 10 min pre-incubation at $0-1^{\circ}$ using $840 \mu \mathrm{M}-\mathrm{LA}$. The esterification rate was determined as the incorporation rate of the pre-existing free ${ }^{14} \mathrm{C}$-labelled $\mathrm{LA}$ into TG at $37^{\circ}$ and was expressed as a percentage. Results are given as means with their standard errors represented by vertical bars for six animals per group. 
However, the capacity of the intestine to convert the absorbed LA to TL at $37^{\circ}$ was moderately reduced in the short-term RF group (Fig. 2), but this difference was not statistically significant.

\section{DISCUSSION}

There was a marked impairment of LA absorption at both high and low concentrations in rats with short-term RF. Earlier studies in normal rats have shown that intestinal transport of essential fatty acids involves two different components. Facilitated transport appears to be the main mechanism of absorption at low lumen concentrations, while passive diffusion is the predominant mechanism for transport at high $(2-10 \mathrm{~mm})$ lumen concentrations. Moreover, the absorptive process is more efficient at low lumen concentrations compared with high concentrations (Chow \& Hollander, 1979a,b). This phenomenon was clearly evident in both the NL control group and animals with short-term RF. The observed depression in intestinal transport at both low and high concentrations of LA in animals with short-term RF suggests that both facilitated and passive components of LA transport are affected by RF. A variety of lumen factors are known to influence the intestinal transport of LA. These include lumen fluid $\mathrm{pH}$, LA concentration, the nature and concentration of the surfactant used as well as the thickness of the unstirred water layer (Chow \& Hollander, 1979a,b). The lumen factors are unlikely to be the cause of the observed difference in LA absorption between the two groups, since identical solutions and experimental conditions were employed. Similarly, no significant difference was noted in the thickness of unstirred water layer between NL and short-term RF animals. Therefore, the observed difference in LA absorption cannot be due to changes in the unstirred water layer.

The present findings indicate that intestinal transport of LA both in vivo and in vitro is impaired in animals with short-term RF. Therefore, the observed abnormality is unlikely to have been due to a rapidly reversible effect of uraemic chemical environment, since the incubations done in vitro were conducted in a physiological solution which was devoid of uraemic chemical compounds. The possible affects of RF on lymphatic or systemic circulations are excluded in the system in vitro and cannot account for the observed impairment of LA uptake in animals with short-term RF. Instead it is considered that an intrinsic mucosal defect is the most likely cause. This defect could be due to a reduction in the total absorptive mucosal surface area (McManus \& Isselbacher, 1970; Powell \& McElveen, 1974) as a part of the overall catabolic effect of uraemia. In fact, a $20-25 \%$ reduction in body-weight and a 30-32\% reduction in intestine weight was observed in animals with short-term RF when compared with the NL control group. In order to examine the possible effect of weight loss and reduced food intake associated with uraemiainduced anorexia, a group of normal rats were pair-fed with their RF counterparts. This led to a comparable reduction in total body and intestine weights in the two groups. Although a mild and statistically non-significant reduction in LA transport was observed in the PF controls, this was not nearly as severe as that seen in animals with short-term RF. Thus, while the observed reduction in the intestinal tissue mass related to decrease in nutrient intake may have played a minor role in the genesis of the observed LA transport defect, other more influential factors must have been involved as well. The studies done in vitro showed a reduction in LA uptake by everted sacs from animals with short-term RF suggesting a possible defect at this level. The marked accumulation of TL in the mucosa of the segments perfused in vivo in the short-term RF animals further suggests the presence of a defect in the transport of esterified LA out of the enterocyte.

Esterification of long-chain fatty acids within the enterocyte is an important step in the intestinal transport of these fatty acids (Marubbio et al. 1974; Ockner \& Isselbacher, 1974). 
The possibility of an esterification defect in animals with short-term RF was investigated by testing the capacity of the intestine to convert absorbed LA to TL at $37^{\circ}$ in vitro. The maximal rate of LA incorporation into TL was found to be moderately reduced in the short-term RF group when compared with the controls. These findings suggest that uraemia-associated impairment of the esterification process may play a role in the genesis of the defective LA transport in animals with short-term RF. These findings are consistent with previous studies (Rodgers et al. 1967; Kessler et al. 1969) that showed a relationship between the degree of inhibition of mucosal esterification and the reduction of long-chain fatty acid absorption in animals with normal kidneys. This point of view is further supported by the observation that incubation at $0^{\circ}$, which reversibly inhibits esterification, obviated the difference in LA uptake between short-term RF and NL controls observed at $37^{\circ}$. It should be noted, however, that the available findings do not allow any conclusion with regard to the possible abnormality of intracellular lipid-carrier proteins in RF, which could potentially produce a similar effect.

In order to determine the effects of duration of RF, a group of animals was studied 5-6 weeks after renal ablation surgery. Surprisingly, in contrast to animals with short-term RF, animals with long-term RF exhibited normal LA absorption in vivo at both low and high concentrations. It thus appears that the effect of RF on LA transport largely depends on the duration of renal insufficiency. It is of interest to note that intestinal morphology is different in animals with different durations of RF. Lohrs et al. (1973) have described denuded intestinal villi in mice with acute RF and increases in villous height in rats with chronic RF. Subsequent studies in this laboratory have confirmed these observations showing increases in villous height, crypt depth and mucosal:serosal length ratio in longterm RF rats when compared with NL and PF controls (Pahl et al. 1990). Hence, it appears that while animals with acute RF demonstrate flattened intestinal villi and reduced surface area for absorption, those with more chronic disease show a restoration of the intestinal architecture, although not to normal levels. It is of interest to speculate that the functional differences found in the present study are at least in part related to the observed morphological changes. Whether or not uraemia alters enterocyte membrane permeability is not known. Further studies are needed to address this issue.

It can be concluded that intestinal absorption of LA is altered in rats with short-term RF but normalizes in animals with long-term RF. Both facilitated and passive transport components are involved. The observed abnormalities seem to be unrelated to uraemiainduced anorexia, reduced food intake or weight loss. Several steps appeared to be affected, including LA uptake by the enterocyte, LA esterification and TL excretion out of the intestinal cell in animals with short-term RF.

\section{REFERENCES}

Anderson, B. W., Levine, A. S., Levitt, D. G., Kneip, J. M. \& Levitt, M. D. (1988). Physiological measurement of luminal stirring in perfused rat jejunum. American Journal of Physiology 254, G843-G848.

Bennet Clark, S., Ekkers, T. E., Singh, A., Balint, J. A., Holt, P. R. \& Rodgers, J. B. Jr (1973). Fat absorption in essential fatty acid deficiency: A model experimental approach to studies of the mechanism of fat malabsorption of unknown etiology. Journal of Lipid Research 14, 581-588.

Brown, J. L. \& Johnston, J. M. (1962). Radioassay of lipid components separated by thin layer chromatography. Journal of Lipid Research 3, 480-481.

Chow, S. L. \& Hollander, D. (1979a). A duel, concentration-dependent absorption mechanism of linoleic acid by rat jejunum in vitro. Journal of Lipid Research 20, 3493356.

Chow, S. \& Hollander, D. (1979 b). Linoleic acid absorption in the unanesthetized rat: Mechanism of transport and influence of luminal factors on absorption. Lipids 14, 378-385.

Delano, B. G., Manis, J. G. \& Manis, T. (1977). Iron absorption in experimental uremia. Nephron 19, $26-31$.

Draper, N. R. \& Smith, J. (1966). Applied Regression Analysis, pp. 1-43. New York: John Wiley \& Sons.

Hollander, D. (1981). Intestinal absorption of vitamin A, E, D and K. Journal of Laboratory Medicine 97 , $449-462$. 
Kates, M. (1975). Techniques of lipidology: Isolation, analysis and identification of lipids. Laboratory Techniques in Biochemistry and Molecular Biology, 2nd ed., vol. 6, pp. 349-350. New York: Elsevier Science Publishing Co.

Kessler, J. I., Mishkin, S. \& Stein, J. (1969). Effect of DL-ethionine on the intestinal absorption and transport of palmitic acid- $1-{ }^{14} \mathrm{C}$ and tripalmitic ${ }^{14} \mathrm{C}$. Role of intramucosal factors in the uptake of luminal lipids. Journal of Clinical Investigation 48, 1397-1407.

Lohrs, U., Castrup, H. J., Wiebecke, B. \& Eder, M. (1973). Effects of experimental uraemia on the small intestinal epithelium. Proceedings of the International Conference on Anatomy, Physiology and Biochemistry of Intestinal Adaptation, pp. 159-169. Stuttgart: Schattauer.

McManus, J. P. A. \& Isselbacher, K. J. (1970). Effect of fasting versus refeeding on the rat small intestine: Morphological, biochemical and functional differences. Gastroenterology 59, 214-221.

Marubbio, A. T. Jr, Morris, J. S. Jr, Bennet Clark, S. \& Holt, P. R. (1974). Monoglyceride modification of jejunal absorption of fatty acid in the rat. Journal of Lipid Research 15, 165-172.

Ockner, R. K. \& Isselbacher, K. J. (1974). Recent concepts of intestinal fat absorption. Review of Physiology, Biochemistry \& Pharmacology 71, 107-146.

Ockner, R. K., Pittman, J. P. \& Yager, J. L. (1972). Differences in the intestinal absorption of saturated and unsaturated long chain fatty acids. Gastroenterology 62, $981-992$.

Pahl, M. V., Erickson, R. A., Vaziri, N. D., Khamiseh, G., Oveisi, F., Jam, K., Seo, J. \& Kutchemeshgi, M. (1990). Intestinal morphometry and bile acid-induced mucosal injury in chronic experimental renal failure. Journal of Laboratory and Clinical Medicine 115, 572-578.

Powell, G. K. \& McElveen, M. A. (1974). Effect of prolonged fasting on fatty acid re-esterification in rat intestinal mucosa. Biochemistry 369, 8-15.

Rodgers, J. B., Riley, E. M., Drummey, G. D. \& Isselbacher, K. J. (1967). Lipid absorption in adrenalectomized rats: The role of altered enzymatic activity in the intestinal mucosa. Gastroenterology 53, 547-556.

Said, H., Vaziri, N. D., Kariger, R. \& Hollander D. (1984). Intestinal absorption of 5-methyltetrahydrofolate in experimental uremia. Acta Vitaminologica et Enzymologica 6, 339-346.

Sterner, G., Lindberg, T. \& Deenneberg, T. (1982). In vivo and in vitro absorption of amino acids and dipeptides in small intestine of uremic rats. Nephron 31, 273-276.

Vaziri, N. D., Hollander, D., Hung, E., Vo, M. \& Dadufalza, L. (1983). Impaired intestinal absorption of vitamin D-3 in azotemic rats. American Journal of Clinical Nutrition 37, 273-276.

Vaziri, N. D., Said, H., Hollander, D., Kariger, R., Patel, N.\& Barbari, A. (1985). Impaired intestinal absorption of riboflavin in experimental uremia. Nephron 41, 26-29.

Vergroesen, A. J. (1977). Physiological effects of dietary linoleic acid. Nutrition Reviews 35, 1-5. 